\title{
A small molecule STAT3 inhibitor, LLL12, enhances cisplatin- and paclitaxel-mediated inhibition of cell growth and migration in human ovarian cancer cells
}

\author{
RUIJIE ZHANG ${ }^{1,2}$, XIANG CHEN ${ }^{2,3}$, SHENGLING FU ${ }^{1,2}$, LIANG XU ${ }^{4}$ and JIAYUH LIN ${ }^{2}$ \\ ${ }^{1}$ Department of Thoracic Surgery, Tongji Hospital, Tongji Medical College, Huazhong University of Science and Technology, \\ Wuhan, Hubei 430030, P.R. China; ${ }^{2}$ Department of Biochemistry and Molecular Biology, University of Maryland School of \\ Medicine, Baltimore, MD 21201; ${ }^{3}$ Department of Pathology and Laboratory Medicine, Weill Cornell Medicine, \\ New York, NY 10021; ${ }^{4}$ Department of Molecular Biosciences, University of Kansas Medical School, \\ University of Kansas, Lawrence, KS 66045, USA
}

Received April 12,2019; Accepted January 17, 2020

DOI: 10.3892/or.2020.7667

\begin{abstract}
Ovarian cancer is one of the most lethal cancer types in American women. The platinum agent cisplatin, and/or paclitaxel, remains the first-line chemotherapy for ovarian cancer, but the treatment success is severely limited by chemoresistance. As previously reported, persistent STAT3 signaling is associated with resistance to cisplatin and paclitaxel. To investigate whether the STAT3 small molecule inhibitor LLL12 can enhance the treatment effect of cisplatin and paclitaxel in ovarian cancer cells, A2780, SKOV3, CAOV-3 and OVCAR5 cells were treated with LLL12, cisplatin and paclitaxel, alone or combination, and cell viability, cell migration, cell growth and protein expression levels were then evaluated. It was found that, for all four human ovarian cancer cell lines, STAT3 phosphorylation was significantly inhibited by LLL12. The combined treatment of LLL12 with paclitaxel or LLL12 with cisplatin exerted significantly greater inhibition of cell viability, cell migration and cell growth than did monotherapy. In addition, LLL12 and cisplatin in combination, or the three drugs in combination, also led to greater inhibition of cell viability and cell migration than combined cisplatin and paclitaxel treatment, a standard treatment for ovarian cancer. The present results demonstrated that the STAT3 small molecule inhibitor LLL12 is a potent inhibitor of STAT3 phosphorylation, cell viability and migration in human ovarian cancer cells. Combining LLL12 with cisplatin or paclitaxel may be a viable therapeutic approach in the treatment of patients with ovarian cancer exhibiting persistent STAT3 signaling.
\end{abstract}

Correspondence to: Professor Jiayuh Lin, Department of Biochemistry and Molecular Biology, University of Maryland School of Medicine, 108 North Greene Street, Baltimore, MD 21201, USA

E-mail: jlin@som.umaryland.edu

Key words: STAT3, ovarian cancer, small molecule inhibitors

\section{Introduction}

Ovarian cancer accounts for $\sim 5 \%$ of global cancer incidence and mortality among women, and is the fifth leading cause of cancer death among American women (1). The standard treatment regimen is cytoreductive surgery followed by a platinum-based regimen of primary chemotherapy (2). This chemotherapy regimen greatly improves patient five-year survival. However, the effect on long-term survival for advanced tumors is not as marked (3-6). The limited response to chemotherapy remains a major obstacle in the treatment of patients with advanced ovarian cancer (3).

For ovarian cancer, chemotherapy with cisplatin and/or paclitaxel remains the first-line treatment. Cisplatin can induce cell apoptosis by interfering with DNA repair mechanisms (7), while paclitaxel promotes the assembly of microtubules and inhibits microtubule depolymerization (8). However, the relapse rate of patients with advanced ovarian cancers is $\sim 70 \%$, due to chemoresistance $(3,4)$. To enhance the chemosensitivity and reduce the recurrence rate, many types of combination therapies have been assessed (7). However, no 'perfect' therapeutic targets have been discovered. Therefore, there is an urgent need to find new therapeutic targets to overcome cisplatin and/or paclitaxel resistance.

As key mediators of cytokine signaling, STAT proteins have become a research focus due to their contribution to oncogenesis (9-12). In many human malignancies, STAT proteins, especially STAT3, are constitutively activated, and the activation rate in ovarian cancer is $94 \%$ (13). Many studies suggest that constitutively activated STAT3 participates in various processes key to malignant progression, such as promoting migration, invasion, cell proliferation, angiogenesis, epithelial-mesenchymal transition (EMT) and metastasis, and it can also induce multidrug chemoresistance (13-18). In particular, cisplatin resistance and paclitaxel resistance has been reported to be associated with the overexpression of STAT3 (13,19-22). Therefore, inhibitors of STAT3 may be used as novel anticancer therapeutics, combined with conventional chemotherapy. 
STAT3 activation is induced by phosphorylation of the tyrosine 705 (Tyr705) residue. A STAT3 inhibitor, LLL12, was previously developed through structure-based drug design. Using Computer models with docking simulation, we found that LLL12 directly binds to the phosphoryl tyrosine 705(pTyr705) binding site (23-24). Numerous experiments have been performed on LLL12, and it can inhibit STAT3 phosphorylation (Tyr705) and STAT3 activity in various human malignant tumors: Multiple myeloma cells, breast cancer cells and pancreatic cancer cells (23-24). STAT3 downstream targets are consequently downregulated to induce cell apoptosis and the inhibition of cell proliferation, migration and colony formation (23-24). However, in ovarian cancer, the function of LLL12 remains unclear.

LLL12 was assayed in four ovarian cancer cell lines (SKOV3, CAOV-3, A2780 and OVCAR5). Although none of these are cisplatin-resistant (or paclitaxel-resistant) ovarian cancer cloned cells, according to the published literature $(25,26)$ and the authors' preliminary results (Figs. S1 and S2), SKOV3 and CAOV-3 cells are more resistant to cisplatin and paclitaxel than A2780 and OVCAR5 cells. In particular, of the high-grade serous human ovarian cancer cell lines, CAOV-3 is considerably more resistant to cisplatin and paclitaxel than OVCAR5.

The present study provides evidence that LLL12 inhibits STAT3 phosphorylation (Tyr705) to decrease the survival of human ovarian cancer cells, and targeting the STAT3 axis may enhance the chemotherapeutic efficiency of cisplatin and paclitaxel in ovarian cancer cells, which may also be key to overcoming cisplatin and paclitaxel resistance.

\section{Materials and methods}

Materials. The STAT3 inhibitor, LLL12, was synthesized by Professor Chenglong Li at the College of Pharmacy, Ohio State University (Columbus, OH, USA). The stock solution was $20 \mathrm{mM}$ (in sterile DMSO) and was stored at $-20^{\circ} \mathrm{C}$.

Cell lines. Human ovarian cancer cell lines (A2780, SKOV3, CAOV-3 and OVCAR5), were purchased from the American Type Culture Collection. A2780, SKOV3 and CAOV-3 were maintained in DMEM (Corning, Inc.; cat. no. 10-013-CV) with $10 \%$ FBS (Sigma-Aldrich; Merck KGaA; cat. no. F2442) and $1 \%$ penicillin/streptomycin (PS); OVCAR5 cells were maintained in RPMI1640 medium (Corning, Inc.; cat. no. $10-040-\mathrm{CV}$ ) with $10 \%$ FBS and $1 \%$ PS. All cell lines were grown in a humidified $37^{\circ} \mathrm{C}$ incubator with $5 \% \mathrm{CO}_{2}$.

Western blot analysis. Ovarian cancer cells were seeded in $10-\mathrm{cm}$ plates at $\sim 70 \%$ cell density. They were treated with LLL12 (A2780: $0.25,0.5$ or $1.0 \mu \mathrm{M}$; SKOV3: $1.0,2.5$ or 5.0 $\mu \mathrm{M}$; CAOV-3: $1.0,2.5$ or $5.0 \mu \mathrm{M}$; and OVCAR5: $0.25,0.5$ or $1.0 \mu \mathrm{M}$ ) or DMSO. Then, the cells were cultured overnight before being harvested for western blot analysis. Ovarian cancer cells were lysed in cold lysis buffer (Cell Lysis Buffer, Cell Signaling Technology, Inc.; 0.5\% 0.2 M PMSF, 0.5\% $0.2 \mathrm{M} \mathrm{NaF}, 0.5 \% 0.2 \mathrm{M} \mathrm{NaPP}, 0.5 \% 0.1 \mathrm{M} \mathrm{Na}_{3} \mathrm{VO}_{4}$ and $4 \% 25 \mathrm{X}$ CP1) and determined using a Microplate BCA Protein Assay kit (Thermo Fisher Scientific, Inc.; cat. no. 23252). Then, equal amounts of proteins ( $40 \mu \mathrm{g}$ ) were separated by $10 \%$ SDS-PAGE.
Proteins were transferred to a PVDF membrane at $350 \mathrm{~mA}$ for $110 \mathrm{~min}$, and then were blocked with $5 \%$ milk for $1 \mathrm{~h}$ at room temperature and incubated with antibodies overnight at $4^{\circ} \mathrm{C}$ : Anti-phosphorylated (p)-STAT3 (Tyr705) (Cell Signaling Technology, Inc.; rabbit mAb; cat. no. NC-CST-9145S), STAT3 (Cell Signaling Technology, Inc.; Rabbit mAb; cat. no. 4904) and GAPDH (Cell Signaling Technology, Inc.; Rabbit mAb; cat. no. 2118S). All primary antibodies were diluted 1:1,000 in 5\% milk. After washing in TBS with Tween-20 three times for $5 \mathrm{~min}$, the membranes were blotted with the horseradish peroxidase-conjugated anti-rabbit IgG secondary antibody diluted 1:10,000 in 5\% milk (Cell Signaling Technology, Inc.; cat. no. 7074) at room temperature for $1.5 \mathrm{~h}$. The p-STAT3 protein were detected by West Femto Maximum Sensitivity Substrate (Thermo Fisher Scientific, Inc.; cat. no. 34096); the STAT3 and GAPDH proteins were detected by enhanced chemiluminescence substrate (PerkinElmer, Inc; cat. no. NEL104001EA). All membranes were scanned with a Storm Scanner (GE Healthcare). The basal level of p-STAT3 in all four cell lines were also tested using the same method.

MTT assay. A2780, SKOV3, CAOV-3 and OVCAR5 cells were seeded in 96-well microtiter plates at a density of 3,000 cells in $100 \mu \mathrm{lmedium} /$ well. On the second day, the cells were treated in each well with LLL12 $(0.1,1.0,0.1$ and $0.01 \mu \mathrm{M}$ for A2780, SKOV3, CAOV-3 and OVCAR5, respectively), cisplatin $(0.5,2.5,2.5$ and $2.5 \mu \mathrm{M}$ for A2780, SKOV3, CAOV-3 and OVCAR5, respectively), paclitaxel $(5,0.25,0.5$ and $0.25 \mu \mathrm{M}$ for A2780, SKOV3, CAOV-3 and OVCAR5, respectively), a combination or the vehicle control (DMSO) at $37^{\circ} \mathrm{C}$. After $72 \mathrm{~h}$, $20 \mu \mathrm{l}$ MTT (Roche Diagnostics) was added to each well. After being incubated for $4 \mathrm{~h}$ at $37^{\circ} \mathrm{C}$, each well was supplemented with $150 \mu \mathrm{l}$ N,N-dimethylformamide (certified ACS; Fisher Chemical; Thermo Fisher Scientific, Inc.) solubilization solution, followed by an incubation overnight protected from light at room temperature. The cell viability was assessed using the absorbance at $595 \mathrm{~nm}$ of each well. The DMSO cells were set at $100 \%$ and the cell viability of drug-treated cells was determined relative to that of DMSO cells. CompuSyn software (www. combosyn.com; 2005 release for Windows) was used to determine the combination index $(\mathrm{CI})$. The $\mathrm{CI}$ value indicates an additive effect when equal to 1 , an antagonistic effect when $>1$, and a synergistic effect when $<1$, based on the theorem of Chou and Talalay (27). The half-maximal inhibitory concentration (IC50) of cisplatin, paclitaxel and LLL12 in the four cell lines was also tested using the MTT assay. The cells were treated with at least five different concentrations of the corresponding drugs and the IC50 was calculated using GraphPad Prism 7 software (GraphPad Software, Inc.).

Wound healing/cell migration assay. A2780 and SKOV3 cells were seeded in 6 -well plates, followed by incubation at $37^{\circ} \mathrm{C}$ overnight. When the cells reached $100 \%$ confluence, the monolayer was scratched using a $100-\mu 1$ pipette tip. After washing with PBS twice, new medium (10\% FBS) with DMSO, or LLL12, cisplatin, paclitaxel or a combination was added to each well. Cells were incubated at $37^{\circ} \mathrm{C}$ until the wounds in the DMSO wells were healed (18 h for SKOV3; $56 \mathrm{~h}$ for A2780). The inhibition of migration was observed by microscope (Nikon TS100 inverted microscope; Nikon Corporation; x10 
magnification) and measured using ImageJ software (National Institutes of Health; version 1.52). The formula for percentage wound healing was as follows: 100-[(final area/initial area) x100\%] (28). An accompanying MTT assay was performed at the same time; 20,000 cells/well were seeded in 96-well plates and treated with the same drugs to evaluate cell viability. The timepoints and the drug concentrations were the same as those for the migration assay.

Cell growth assay. For the cell growth assays, A2780, SKOV3, CAOV-3 and OVCAR5 cells were seeded in 12-well plates. The cell density was dependent on the growth ability of the cells (A2780: $0.5 \times 10^{4}$ cells/well; SKOV3: $1 \times 10^{4}$ cells/well; CAOV-3: $2.5 \times 10^{4}$ cells/well; and OVCAR5: $1 \times 10^{4}$ cells/well). Then, the cells were treated with DMSO $(1 \mu \mathrm{l})$, or LLL12, cisplatin, paclitaxel or a combination. A2780 cells were treated with DMSO, or LLL12 $(0.05 \mu \mathrm{mol} / \mathrm{l})$, cisplatin $(0.25 \mu \mathrm{mol} / \mathrm{l})$, paclitaxel $(10 \mathrm{nmol} / \mathrm{l})$ or a combination. SKOV3 cells were treated with DMSO, or LLL12 $(0.25 \mu \mathrm{mol} / \mathrm{l})$, cisplatin $(0.5 \mu \mathrm{mol} / \mathrm{l})$, paclitaxel $(10 \mathrm{nmol} / \mathrm{l})$ or a combination. CAOV-3 cells were treated with DMSO, or LLL12 $(0.05 \mu \mathrm{mol} / \mathrm{l})$, cisplatin $(0.25 \mu \mathrm{mol} / \mathrm{l})$, paclitaxel $(10 \mathrm{nmol} / \mathrm{l})$ or a combination. OVCAR5 cells were treated with DMSO, or LLL12 $(0.05 \mu \mathrm{mol} / \mathrm{l})$, cisplatin $(0.25 \mu \mathrm{mol} / \mathrm{l})$, paclitaxel $(10 \mathrm{nmol} / \mathrm{l})$ or a combination. Cell numbers were counted on days 2, 4, 6 and 8 after treatment. Then, growth curves were drawn and the cell numbers on day 8 were compared.

Statistical analysis. Significance was determined using GraphPad Prism 7 software (GraphPad Software Inc.). The data are expressed as the mean \pm SD from three repeats. Oneand two-way ANOVA with Tukey's test was used to analyze the statistical differences between groups. $\mathrm{P}<0.05$ was considered to indicate a statistically significant difference.

\section{Results}

STAT3 phosphorylation is inhibited by LLL12 in ovarian cancer cells. To examine the ability of LLL12 to inhibit STAT3 phosphorylation (Tyr705), western blot analysis was performed in ovarian cancer cells (A2780, SKOV3, CAOV-3 and OVCAR5). Compared with DMSO control, the p-STAT3 expression in A2780, SKOV3, CAOV-3 and OVCAR5 ovarian cancer cell lines was decreased after treatment with LLL12 (0.25-1.0 $\mu \mathrm{M})$ (Fig. 1). Compared with cisplatin or paclitaxel alone, the combination of LLL12 with cisplatin or/and paclitaxel led to a greater inhibitory effect on p-STAT3. These results confirmed that in human ovarian cancer cell lines, LLL12 is a potent STAT3 inhibitor.

LLL12 combined with cisplatin or paclitaxel synergistically inhibits the cell viability of ovarian cancer cells. The first line chemotherapy for ovarian cancer is cisplatin combined with paclitaxel. Therefore, the present study first investigated whether LLL12 and cisplatin or paclitaxel could generate a more potent suppression of cell viability than any single agent alone in ovarian cancer cells. All four ovarian cancer cell lines were treated with different concentrations of LLL12, cisplatin, paclitaxel or a combination. After $72 \mathrm{~h}$ of treatment, the combination treatment of LLL12 with cisplatin or paclitaxel induced greater inhibition of cell viability than that of any monotherapy (Fig. 2). The CI values were $<1$ for all the combination treatments, which indicated that the cell viability of ovarian cancer cells was synergistically inhibited by LLL12 combined with paclitaxel. Compared with the standard ovarian cancer combination treatment of cisplatin with paclitaxel, the combination treatment of LLL12 with cisplatin or/and paclitaxel also generated greater inhibitory effects on cell viability, and the combination of the three drugs elicited the greatest inhibitory effects (Fig. 2).

LLL12 combined with cisplatin inhibits the cell migration of ovarian cancer cells. For tumor invasion and metastasis, cell migration is an important process. Previous studies have indicated that the inhibition of STAT3 may reduce cancer cell migration (16-18). Therefore, the present study evaluated the effect of LLL12 combined with cisplatin, compared with a paclitaxel and cisplatin combination, on cell migration. Representative images are shown in Figs. 3 and 4. The A2780 cells were treated with different drugs for $60 \mathrm{~h}$ until the wound in the DMSO control group was healed; the combined LLL12 and cisplatin treatment had a more significant inhibitory effect on wound healing/cell migration than either monotherapy (Fig. 3). Compared with the combination treatment of cisplatin with paclitaxel, the combination treatment of LLL12 with cisplatin or/and paclitaxel generated a more significant inhibitory effect on cell migration, and the combination of the three drugs elicited the greatest inhibitory effect (Fig. 3). Consistently, in SKOV3 ovarian cancer cells, the combined LLL12 and cisplatin or/and paclitaxel treatment also exerted a greater inhibitory effect on cell migration than the monotherapies or the paclitaxel and cisplatin combination, and the combination of the three drugs elicited the greatest inhibitory effect (Fig. 4). The accompanying MTT assay was performed at the same time $(20,000$ cells/well were seeded in 96-well plates, then treated with the same drugs to evaluate cell viability; the timepoints and the drug concentrations were the same as those of the migration assay), and there were no significant differences in cell viability between the groups. This indicated that the inhibition of cell proliferation had little effect on this experiment.

LLL12 combined with cisplatin inhibits the cell growth of ovarian cancer cells. It was demonstrated that LLL12 synergistically inhibited the cell viability of ovarian cancer cells in combination with cisplatin and paclitaxel. The present study further evaluated the effect of LLL12 on cell growth combined with cisplatin or paclitaxel. The results showed that LLL12 inhibited cell growth in all four ovarian cancer lines (Fig. 5). The combined treatment of LLL12 with cisplatin had a more significant inhibitory effect on cell growth than either monotherapy in all four human ovarian cancer cell lines (Fig. 5A). Furthermore, the LLL12 and paclitaxel combination exerted a greater inhibitory effect on cell growth than either single agents alone in all four ovarian cancer cell lines (Fig. 5B).

\section{Discussion}

Ovarian cancer is the leading cause of cancer-associated death in women in the US (1). Most patients are diagnosed at 


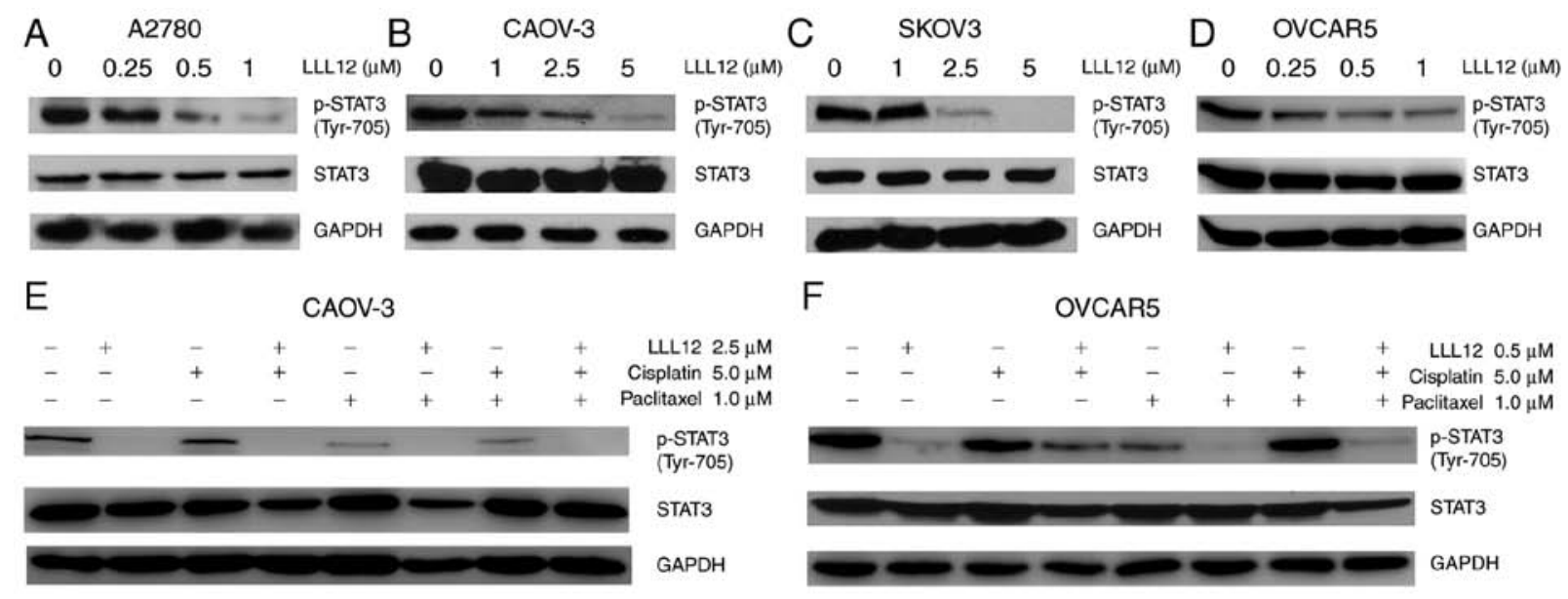

Figure 1. LLL12 inhibits p-STAT3 in human ovarian cancer cells. Ovarian cancer cell lines were treated with LLL12, and the levels of STAT3 phosphorylation were decreased: (A) A2780; (B) CAOV-3; (C) SKOV3; and (D) OVCAR5. Compared with cisplatin or paclitaxel alone, the combination of LLL12 with cisplatin or/and paclitaxel appeared to have a greater inhibitory effect on p-STAT3 in (E) CAOV-3 and (F) OVCAR5 cells. p, phosphorylated.
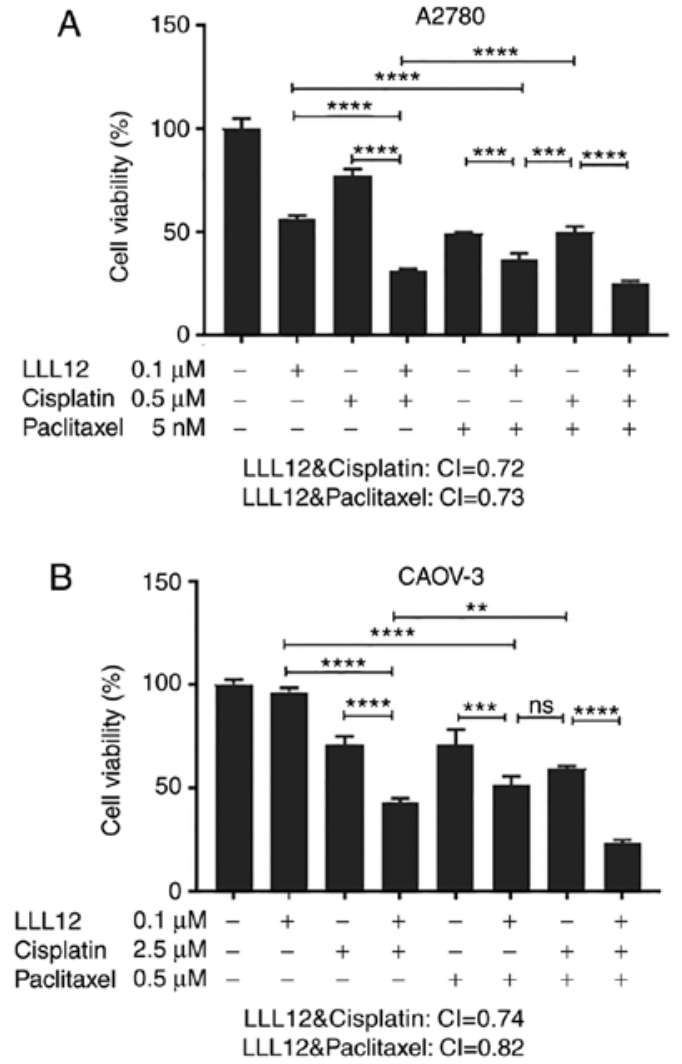
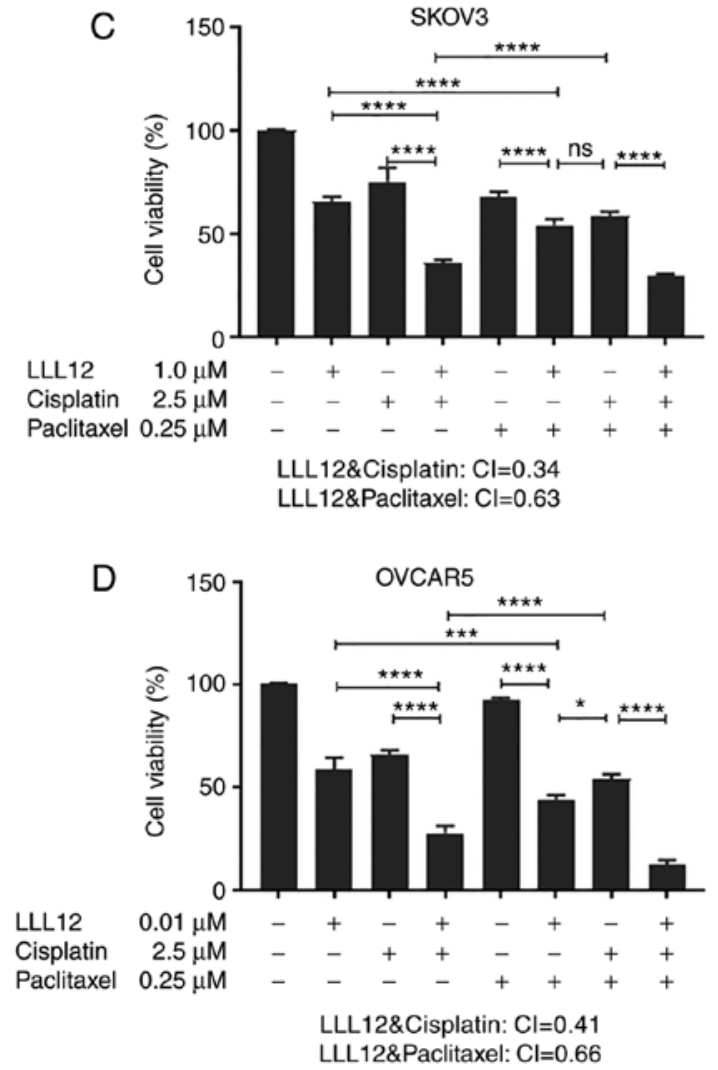

Figure 2. LLL12 combined with cisplatin or paclitaxel synergistically inhibits the cell viability of ovarian cancer cells. Cells were seeded in 96-well plate at a density of 3,000 cells per well. Each well was treated with DMSO, or LLL12, cisplatin, paclitaxel or a combination for $72 \mathrm{~h}$. An MTT assay was performed to evaluate cell viability. (A) A2780; (B) CAOV-3; (C) SKOV3; and (D) OVCAR5. ${ }^{*} \mathrm{P}<0.05,{ }^{* * *} \mathrm{P}<0.01,{ }^{* * * *} \mathrm{P}<0.001$ and ${ }^{* * * * *} \mathrm{P}<0.0001$. CI, combination index; ns, not significant.

a late stage, due to the lack of specific symptoms associated with early-stage ovarian cancer. Standardized treatment for late-stage ovarian cancers is cytoreductive surgery followed by a platinum-based regimen of primary chemotherapy (3). Cisplatin combined with paclitaxel is recognized as the first-line chemotherapy for advanced ovarian cancer worldwide, despite severe side effects (2). Although the treatment regimen is effective and no adequate replacement has yet been found,
$75 \%$ of patients experience recurrence of ovarian cancer (7), and data show that the 5-year survival rate of patients with ovarian cancer has remained almost unchanged after diagnosis in the past 20 years $(1,26)$. There are many reasons for cancer recurrence, among which chemoresistance to cisplatin and/or paclitaxel is the most commonly reported $(29,30)$. Therefore, there is a need to discover new therapeutic targets and new drugs to enhance the cisplatin- and paclitaxel-mediated inhibitory 

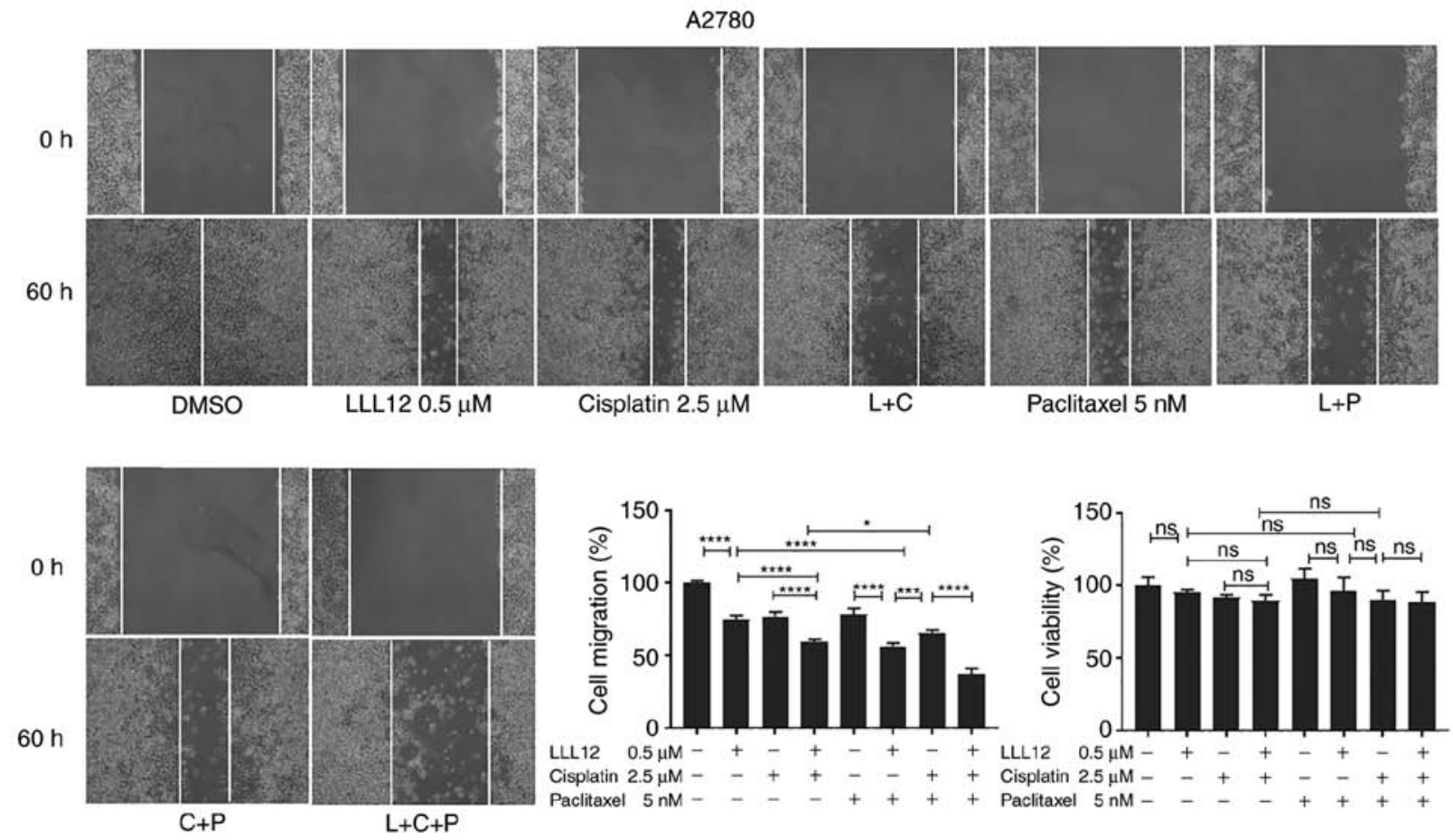

Figure 3. LLL12 combined with cisplatin inhibits the cell migration of A2780 ovarian cancer cells. A wound healing assay for migration was performed to evaluate the migration capability of A2780 cells treated with LLL12 $(0.5 \mu \mathrm{mol} / \mathrm{l})$, cisplatin $(2.5 \mu \mathrm{mol} / \mathrm{l})$, paclitaxel $(5 \mathrm{nmol} / \mathrm{l})$ or a combination, or the vehicle (DMSO). Magnification, x100. An MTT assay was also performed: 20,000 cells/well were seeded in 96-well plates, then treated with the same drugs to evaluate cell viability. The timepoints and the drug concentrations were the same as those for the migration assay. ${ }^{*} \mathrm{P}<0.05,{ }^{* * * *} \mathrm{P}<0.001$ and ${ }^{* * * *} \mathrm{P}<0.0001$. L, LLL12; C, cisplatin; P, paclitaxel; ns, not significant.

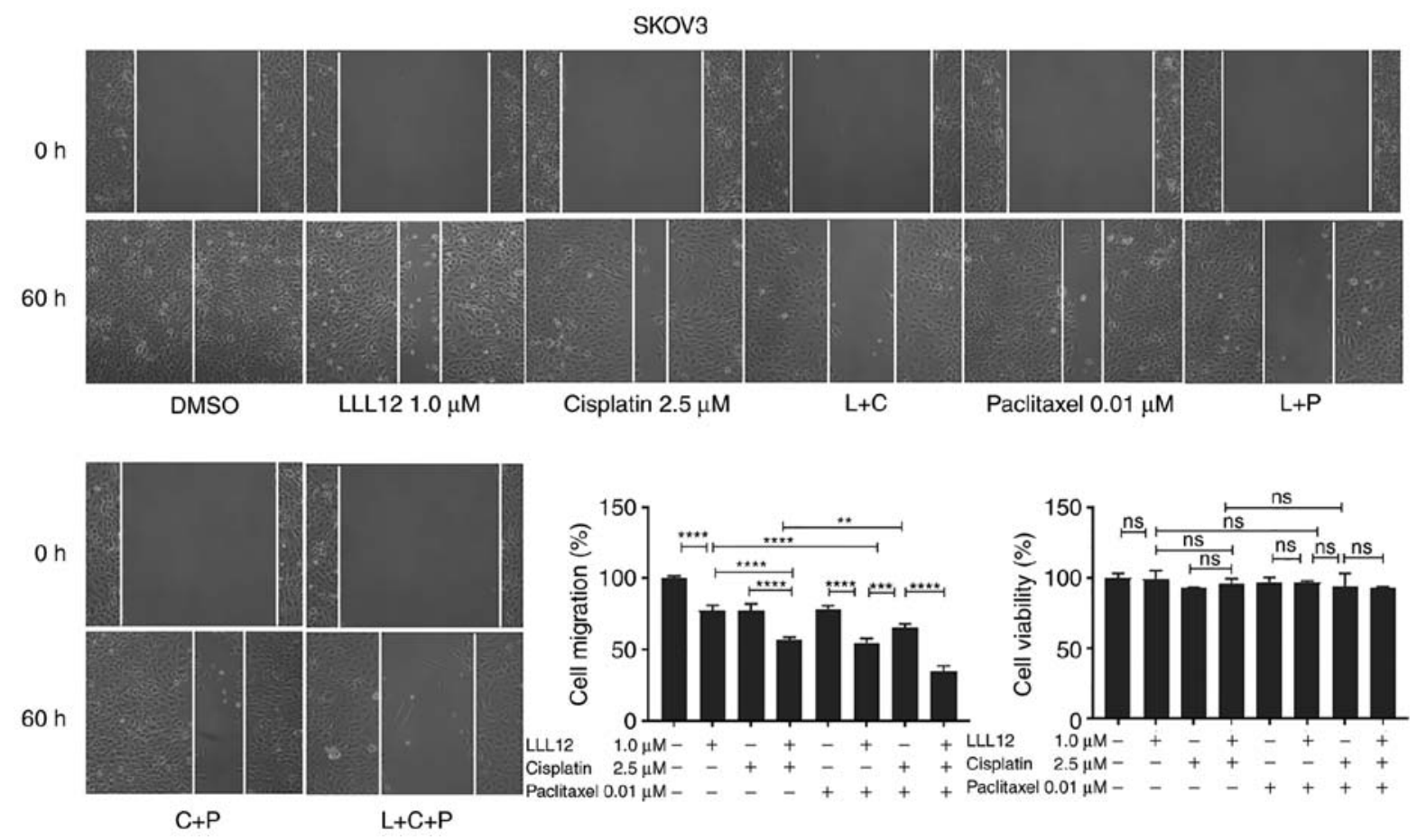

Figure 4. LLL12 combined with cisplatin inhibits the cell migration of SKOV-3 ovarian cancer cells. A wound healing assay for migration was performed to evaluate the migration capability of SKOV3 cells treated with LLL12 $(1.0 \mu \mathrm{mol} / \mathrm{l})$, cisplatin $(2.5 \mu \mathrm{mol} / \mathrm{l})$, paclitaxel $(10 \mathrm{nmol} / \mathrm{l})$ or a combination, or the vehicle (DMSO). Magnification, x100. An MTT assay was also performed: 20,000 cells/well were seeded in 96-well plates, then treated with the same drugs to evaluate cell viability. The timepoints and the drug concentrations were the same as those for the migration assay. ${ }^{* * *} \mathrm{P}<0.01,{ }^{* * * *} \mathrm{P}<0.001$ and ${ }^{* * * * *} \mathrm{P}<0.0001$. L, LLL12; C, cisplatin; P, paclitaxel; ns, not significant.

effect on ovarian cancer. Chemoresistance is multifactorial and complex. Many studies have been performed to explore the gene signatures associated with chemoresistance (30-32), and no consistent results have been found. 

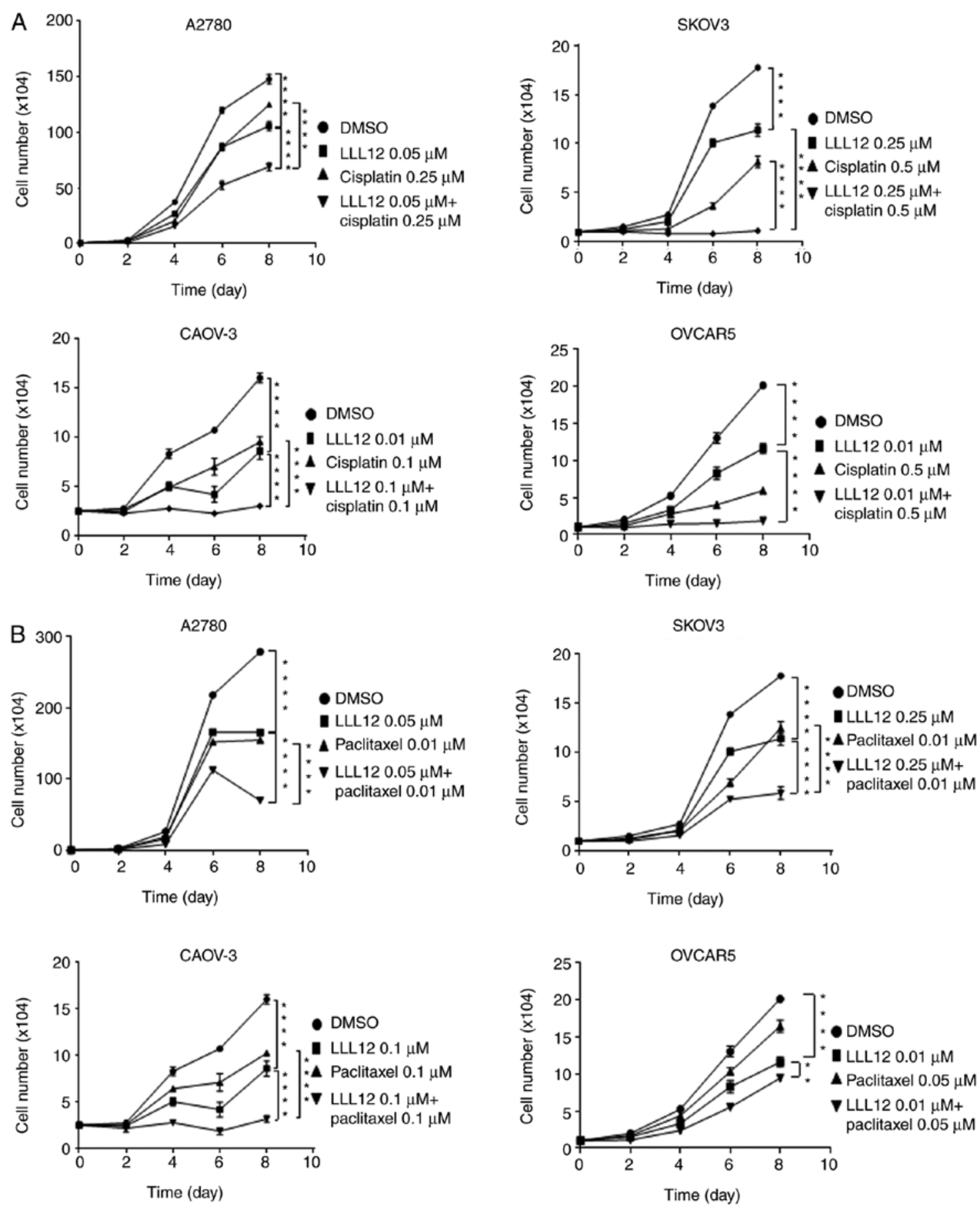

Figure 5. LLL12 combined with cisplatin and/or paclitaxel inhibits the cell growth of ovarian cancer cells. Ovarian cancer cells were seeded in 12-well plates, and treated with DMSO, or (A) LLL12, cisplatin or a combination of the two; or (B) LLL12, paclitaxel or a combination of the two. Growth curves were drawn according to the cell numbers on days $2,4,6$ and 8 after treatment, and the cell numbers on day 8 were compared. ${ }^{* *} \mathrm{P}<0.01$ and ${ }^{* * * * *} \mathrm{P}<0.0001$ on day 8 .

As reported, STAT proteins have become a research focus due to their contribution to oncogenesis (9-12). In numerous human malignancies, STAT proteins, especially STAT3, are constitutively activated, and the activation rate in ovarian cancer is $94 \%$ (13). Studies have reported that constitutive activation of STAT3 is involved in various processes during malignant progression, including migration, invasion, cell proliferation, angiogenesis, EMT and metastasis, and it may also induce multidrug chemoresistance (13-18). In particular, cisplatin and paclitaxel resistance has also been reported to be associated with the overexpression of STAT3 $(13,19-22)$; thus, inhibitors of STAT3 may be used as novel anticancer therapeutics, in combination with conventional chemotherapy.

A previous study demonstrated that constitutive activation of STAT3 is present in ovarian cancer cell lines, but not in normal ovarian surface epithelial cells (33). Many studies have shown that the STAT3 pathway is often overexpressed and activated in cisplatin- and/or paclitaxel-resistant ovarian cancer cells, as compared with paired parental cell lines that are cisplatin- and/or paclitaxel-sensitive (16,17,19-21). Inhibition of STAT3 enhances apoptosis induced by cisplatin and paclitaxel in ovarian cancer cells $(33,34)$. Furthermore, 
silencing of the STAT3 signaling pathway can reverse the inherent and induced chemoresistance of human ovarian cancer cells (35). It appears that STAT3 inhibitors may be important for combatting chemoresistance to cisplatin and paclitaxel. Many peptide-based inhibitors of STAT3 have been synthesized and reported, but common problems are poor cell permeability and in vivo stability, which have limited their use $(36,37)$. In addition, nonpeptide small molecule inhibitors of STAT3 have also been synthesized, such as SD-1029 (38) Stattic (39), STA-21 (40), S3I-201 (41) and WP1066 (42) among others.

In order to solve the problems mentioned above, LLL12, a small molecule STAT3 inhibitor, was synthesized (24). According to previous work, LLL12 can inhibit STAT3 phosphorylation in many cancer cells, such as glioblastoma, pancreatic cancer, multiple myeloma and breast cancer cells $(23,24,43-46)$. Furthermore, LLL12 has good cell permeability and stability in vivo, and demonstrated marked inhibition of STAT3 phosphorylation in an animal model $(23,24,45)$. Compared with STAT3 inhibitors reported previously, namely S3I-201 and WP1066, LLL12 was demonstrated to be more potent in the inhibition of cancer cell viability (43). It was also demonstrated that LLL12 had a minimal apoptotic effect on normal human cells $(23,24)$. Therefore, LLL12 was assessed in ovarian cancer cells. STAT3 phosphorylation was decreased by LLL12 in all four ovarian cancer cells. Cell viability, cell migration and cell growth assays were performed; the results showed that, compared with monotherapies, the combination of LLL12 and cisplatin or paclitaxel led to more potent inhibition. Simultaneously, it was found that the combination of cisplatin with LLL12 led to more effective inhibition of cell viability and cell migration than that of cisplatin with paclitaxel. The cell migration assay was trialed under serum-starvation conditions, but the cells of the DMSO control group died before the wound healed. Therefore, $10 \%$ serum was used in this assay. CAOV-3 and OVCAR5 cells were not tested in the wound healing/cell migration assays, because their growth phenotype in a monolayer is not suitable for wound healing/cell migration assays. The cell density for the cell growth assay was much lower than that used for the western blot analysis; therefore, low doses of LLL12 with cisplatin or paclitaxel were used. If a higher dose of LLL12 is used, it alone could cause an almost complete inhibition of cell growth, and therefore, it would not be possible to verify whether LLL12 in combination with cisplatin or paclitaxel could exert stronger inhibition than either single agent alone.

Of the four cell lines, OVCAR-5 is a human epithelial carcinoma cell line of the ovary, established from the ascitic fluid of a patient with progressive ovarian adenocarcinoma without prior cytotoxic treatment; this cell line had been used as high-grade serous human ovarian cancer cell line since 1984 (47). However, there was a study published in 2016 that provided some evidence that OVCAR-5 might be regarded as non-ovarian, being gastrointestinal in origin (48). An STR profile analysis was performed for the OVCAR5 cells. The results demonstrated that the sample OVCAR5 cells shared 14 out of 14 alleles (100\%) with the American Type Culture Collection reference. Additionally, many studies have used OVCAR5 as an ovarian cancer cell line since 20016 (49-51), so the experimental data for this cell line was retained in the present study.

From the literatures, there are ovarian cancer cell that were selected by gradually increasing the concentration of cisplatin, and cloning the surviving cells as cisplatin-resistant cells. However, such cisplatin- or paclitaxel-resistant cell lines were unavailable for the present study. Nevertheless, the SKOV3 and CAOV-3 ovarian cancer cell lines are more resistant to cisplatin and paclitaxel than the A2780 and OVCAR5 ovarian cancer cell lines, based on published literature $(25,26)$ and the authors' preliminary results. In particular, among high-grade serous human ovarian cancer cell lines, CAOV-3 is much more resistant to cisplatin or paclitaxel than OVCAR5. Notably, the basal levels of p-STAT3 (Fig. S3) and the IC50 of LLL12 (Fig. S4) were also higher in the SKOV3 and CAOV-3 ovarian cancer cell lines. These results further supported the hypothesis that levels of p-STAT3 are positively associated with resistance to cisplatin and paclitaxel. The results presented in this manuscript showed that LLL12 may enhance the cisplatin- and paclitaxel-mediated inhibition of cell growth and migration in the human ovarian cancer cell lines SKOV3 and CAOV-3, which are resistant to cisplatin or/and paclitaxel.

In conclusion, the present findings suggested LLL12 may enhance the cisplatin- and paclitaxel-mediated inhibition of cell growth and migration in human ovarian cancer cells. This may be key to combatting cisplatin and paclitaxel resistance, which requires further research. Translating these findings to the clinical realm will require further investigation ensure the safety of this potential treatment.

\section{Acknowledgements}

The authors would like to thank Dr Rich Eckert at the Department of Biochemistry and Molecular Biology, University of Maryland (Baltimore, MD, USA) for providing the microscope used to evaluate the wound healing assay.

\section{Funding}

This research was supported by the University of Maryland School of Medicine and the Comprehensive Cancer Center start up fund.

\section{Availability of data and materials}

All data generated or analyzed during this study are included in this published article.

\section{Authors' contributions}

JL acquired funding and resources. RZ, XC, SF, LX and $\mathrm{JL}$ contributed to the design of the study. RZ, XC, SF and LX acquired and analyzed the data. RZ and JL drafted the manuscript. RZ, XC, SF, LX and JL revised and edited the manuscript. All authors read and approved the final version of the manuscript.

\section{Ethics approval and consent to participate}

Not applicable. 


\section{Patient consent for publication}

Not applicable.

\section{Competing interests}

The authors declare that they have no competing interests.

\section{References}

1. Siegel RL, Miller KD and Jemal A: Cancer statistics, 2017. CA Cancer J. Clin 67: 7-30, 2017.

2. Mei L, Chen H, Wei DM, Fang F, Liu GJ, Xie HY, Wang X, Zou J, Han X and Feng D: Maintenance chemotherapy for ovarian cancer. In The Cochrane Library; John Wiley, Sons, Hoboken, NJ, USA, 2013

3. Dressman HK, Berchuck A, Chan G, Zhai J, Bild A, Sayer R, Cragun J, Clarke J, Whitaker RS, Li L, et al: An integrated genomic-based approach to individualized treatment of patients with advanced-stage ovarian cancer. J Clin Oncol 25: 517-525, 2007.

4. Jazaeri AA, Awtrey CS, Chandramouli GV, Chuang YE, Khan J, Sotiriou C, Aprelikova O, Yee CJ, Zorn KK, Birrer MJ, et al: Gene expression profiles associated with response to chemotherapy in epithelial ovarian cancers. Clin Cancer Res 11: 6300-6310, 2005.

5. Sudo T: Molecular-targeted therapies for ovarian cancer: Prospects for the future. Int J Clin Oncol 17: 424-429, 2012.

6. Sueblinvong T, Ghebre R, Iizuka Y, Pambuccian SE, Isaksson Vogel R, Skubitz AP and Bazzaro M: Establishment, characterization and downstream application of primary ovarian cancer cells derived from solid tumors. PLOS One 7: e50519, 2012.

7. Dasari S and Tchounwou PB: Cisplatin in cancer therapy: Molecular mechanisms of action. Eur J Pharmacol 740: 364-378, 2014.

8. Beth A: Weaver How Taxol/paclitaxel kills cancer cells. Mol Biol Cell 25: 2677-2681, 2014

9. Buettner R, Mora LB and Jove R: Activated STAT signaling in human tumors provides novel molecular targets for therapeutic intervention. Clin Cancer Res 8: 945-954, 2002.

10. Bromberg J and Darnell JE Jr: The role of STATs in transcriptional control and their impact on cellular function. Oncogene 19 2468-2473, 2000.

11. Turkson $\mathbf{J}$ and Jove R: STAT proteins: Novel molecular targets for cancer drug discovery. Oncogene 19: 6613-6626, 2000

12. Bowman T, Garcia R, Turkson J and Jove R: STATs in oncogenesis. Oncogene 19: 2474-2488, 2000.

13. Duan Z, Foster R, Bell DA, Mahoney J, Wolak K, Vaidya A Hampel C, Lee H and Seiden MV: Signal transducers and activators of transcription 3pathway activation in drug-resistant ovarian cancer. Clin Cancer Res 12: 5055-5063, 2006.

14. Silver DL, Naora H, Liu J, Cheng W and Montell DJ: Activated signal transducer and activator of transcription (STAT) 3: Localization in focal adhesions and function in ovarian cancer cell motility. Cancer Res 64: 3550-3558, 2004.

15. Rosen DG, Mercado-Uribe I, Yang G, Bast RC Jr, Amin HM, Lai R and Liu J: The role of constitutively active signal transducer and activator of transcription 3 in ovarian tumorigenesis and prognosis. Cancer 107: 2730-2740, 2006.

16. Yue P, Zhang X, Paladino D, Sengupta B, Ahmad S, Holloway RW, Ingersoll SB and Turkson J: Hyperactive EGF receptor, Jaks and Stat 3 signaling promote enhanced colony-forming ability, motility and migration of cisplatin-resistant ovarian cancer cells. Oncogene 31: 2309-2322, 2012.

17. Kandala PK and Srivastava SK: Diindolylmethane suppresses ovarian cancer growth and potentiates the effect of cisplatin in tumor mouse model by targeting signal transducer and activator of transcription 3 (STAT3). BMC Med 10: 9, 2012

18. Colomiere M, Findlay J, Ackland L and Ahmed N: Epidermal growth factor-induced ovarian carcinoma cell migration is associated with JAK2/STAT3 signals and changes in the abundance and localization of alpha6beta1 integrin. Int J Biochem Cell Biol 41: 1034-1045, 2009.

19. Duan Z, Foster R, Bell DA, Mahoney J, Wolak K, Vaidya A, Hampel C, Lee H and Seiden MV: Signal transducers and activators of transcription 3 pathway activation in drug-resistant ovarian cancer. Clin Cancer Res 12: 5055-5063, 2006.
20. Duan Z, Ames RY, Ryan M, Hornicek FJ, Mankin H and Seiden MV: CDDO-Me, a synthetic triterpenoid, inhibits expression of IL-6 and Stat 3 phosphorylation in multi-drug resistant ovarian cancer cells. Cancer Chemother Pharmacol 63: 681-689, 2009.

21. Zhang X, Liu P, Zhang B, Wang A and Yang M: Role of STAT3 decoy oligodeoxynucleotides on cell invasion and chemosensitivity in human epithelial ovarian cancer cells. Cancer Genet Cytogenet 197: 46-53, 2010.

22. Sheng WJ, Jiang H, Wu DL and Zheng JH: Early responses of the STAT3 pathway to platinum drugs are associated with cisplatin resistance in epithelial ovarian cancer. Braz J Med Biol Re 46: 650-658, 2013

23. Lin L, Benson DM Jr, DeAngelis S, Bakan CE, Li PK, Li C and Lin J: A small molecule, LLL12 inhibits constitutive STAT3 and IL-6 induced STAT3 signaling and exhibits potent growth suppressive activity in human multiple myeloma cells. Int J Cancer 130: 1459-1469, 2012

24. Lin L, Hutzen B, Li PK, Ball S, Zuo M, DeAngelis S, Foust E, Sobo M, Friedman L, Bhasin D, et al: A Novel Small Molecule, LLL12, Inhibits STAT3 phosphorylation and activities and exhibits potent growth-suppressive activity in human cancer cells. Neoplasia 12: 39-50, 2010.

25. Yang J, Zaman MM, Vlasakov I, Roy R, Huang L, Martin CR, Freedman SD, Serhan CN and Moses MA: Adipocytes promote ovarian cancer chemoresistance. Sci Rep 9: 13316, 2019.

26. Ma S, Tan W, Du B, Liu W, Li W, Che D and Zhang G: Oridonin effectively reverses cisplatin drug resistance in human ovarian cancer cells via induction of cell apoptosis and inhibition of matrix metalloproteinase expression. Mol Med Rep 13: 3342-3348, 2016.

27. Chou TC: Theoretical basis, experimental design, and computerized simulation of synergism and antagonism in drug combination studies. Pharmacol Rev 58: 621-681, 2006.

28. Fu S, Chen X, Lin HJ and Lin J: Inhibition of interleukin 8/CXC chemokine receptor $1 / 2$ signaling reduces malignant features in human pancreatic cancer cells. Int J Oncol 53: 349-357, 2018.

29. Cannistra SA: Cancer of the ovary. N Engl J Med 351: 2519-2529, 2004.

30. Bristow RE and Chi DS: Platinum-based neoadjuvant chemotherapy and interval surgical cytoreduction for advanced ovarian cancer: A meta-analysis. Gynecol Oncol 103: 1070-1076, 2006.

31. Arora S, Bisanz KM, Peralta LA, Basu GD, Choudhary A, Tibes R and Azorsa DO: RNAi screening of the kinome identifies modulators of cisplatin response in ovarian cancer cells Gynecol Oncol 118: 220-227, 2010.

32. Kang J, D'Andrea AD and Kozono D: A DNA repair pathway-focused score for prediction of outcomes in ovarian cancer treated with platinum-based chemotherapy. J Natl Cancer Inst 104: 670-681, 2012

33. Huang M, Page C, Reynolds RK and Lin J: Constitutive activation of stat 3 oncogene product in human ovarian carcinoma cells. Gynecol Oncol 79: 67-73, 2000.

34. Burke WM, Jin X, Lin HJ, Huang M, Liu R, Reynolds RK and Lin J: Inhibition of constitutively active Stat 3 suppresses growth of human ovarian and breast cancer cells. Oncogene 20: 7925-7934, 2001

35. Han Z, Feng J, Hong Z, Chen L, Li W, Liao S, Wang X, Ji T, Wang S, Ma D, et al: Silencing of the STAT3 signaling pathway reverses the inherent and induced chemoresistance of human ovarian cancer cells. Biochem Biophys Res Commun 435: 188-194, 2013.

36. Turkson J, Ryan D, Kim JS, Zhang Y, Chen Z, Haura E, Laudano A, Sebti S, Hamilton AD and Jove R: Phosphotyrosyl peptides block Stat3-mediated DNA binding activity, gene regulation, and cell transformation. J Biol Chem 276: 45443-45455, 2001.

37. Burke TR Jr, YaoZJ,Liu DG, Voigt J and Gao Y: Phosphoryltyrosyl mimetics in the design of peptide-based signal transduction inhibitors. Biopolymers 60: 32-44, 2001.

38. Iwamaru A, Szymanski S, Iwado E, Aoki H, Yokoyama T, Fokt I, Hess K, Conrad C, Madden T, Sawaya R, et al: A novel inhibitor of the STAT3 pathway induces apoptosis in malignant glioma cells both in vitro and in vivo. Oncogene 26: 2435-2444, 2007.

39. Siddiquee K, Zhang S, Guida WC, Blaskovich MA, Greedy B, Lawrence HR, Yip ML, Jove R, McLaughlin MM, Lawrence NJ, et al: Selective chemical probe inhibitor of Stat3, identified through structure-based virtual screening, induces antitumor activity. Proc Natl Acad Sci USA 104: 7391-7396, 2007. 
40. Song H, Wang R, Wang S and Lin J: A low-molecular-weight compound discovered through virtual database screening inhibits Stat 3 function in breast cancer cells. Proc Natl Acad Sci USA 102: 4700-4705, 2005.

41. Schust J, Sperl B, Hollis A, Mayer TU and Berg T: Stattic: A small molecule inhibitor of STAT3 activation and dimerization. Chem Biol 13: 1235-1242, 2006.

42. Duan Z, Bradner JE, Greenberg E, Levine R, Foster R, Mahoney J and Seiden MV: SD-1029 inhibits signal transducer and activator of transcription 3nuclear translocation. Clin Cancer Res 12: 6844-6852, 2006.

43. Wei CC, Ball S, Lin L, Liu A, Fuchs JR, Li PK, Li C and Lin J: Two small molecule compounds, LLL12 and FLLL32, exhibit potent inhibitory activity on STAT3 in human rhabdomyosarcoma cells. Int J Oncol 38: 279-285, 2011.

44. Onimoe GI, Liu A, Lin L, Wei CC, Schwartz EB, Bhasin D, Li C Fuchs JR, Li PK, Houghton P, et al: Small molecules, LLL12 and FLLL32, inhibit STAT3and exhibit potent growth suppressive activity in osteosarcoma cells and tumor growth in mice. Invest New Drugs 30: 916-926, 2012.

45. Ball S, Li C, Li PK and Lin J: The Small Molecule, LLL12, Inhibits STAT3 Phosphorylation and Induces Apoptosis in Medulloblastoma and Glioblastoma Cells. PLoS One 6: e18820, 2011.
46. Couto JI, Bear MD, Lin J, Pennel M, Kulp SK, Kisseberth WC and London CA: Biologic activity of the novel small molecule STAT3 inhibitor LLL12 against canine osteosarcoma cell lines. BMC Vet Res 8: 244, 2012.

47. Hamilton TC, Young RC and Ozols RF: Experimental model systems of ovarian cancer: Applications to the design and evaluation of new treatment approaches. Semin Oncol 11: 285-298, 1984

48. Blayney JK, Davison T, McCabe N, Walker S, Keating K, Delaney T, Greenan C, Williams AR, McCluggage WG, Capes-Davis A, et al: Prior knowledge transfer across transcriptional data sets and technologies using compositional statistics yields new mislabelled ovarian cell line. Nucleic Acids Res 44: e137, 2016.

49. Bratcher NA, Frost DJ, Hickson J, Huang X, Medina LM, Oleksijew A, Ferguson DC and Bolin S: Effects of buprenorphine in a preclinical orthotopic tumor model of ovarian carcinoma in female CB17 SCID Mice. J Am Assoc Lab Anim Sci 58: 583-588, 2019.

50. Capo-Chichi CD, Cai KQ and Xu XX: Overexpression and cytoplasmic localization of caspase- 6 is associated with lamin A degradation in set of ovarian cancers. Biomark Res 6:30, 2018.

51. Fang Z, Li T, Chen W, Wu D, Qin Y, Liu M, Wu G, He L, Li H and $\mathrm{Gu} \mathrm{H}$ : Gab2 promotes cancer stem cell like properties and metastatic growth of ovarian cancer via downregulation of miR-200c. Exp Cell Res 382: 111462, 2019. 